\title{
Instanton-dyon ensembles reproduce deconfinement and chiral restoration phase transitions
}

\author{
Edward Shuryak* \\ ${ }^{1}$ Department of Physics and Astronomy, Stony Brook University, \\ Stony Brook NY 11794 USA
}

\begin{abstract}
Paradigm shift in gauge topology at finite temperatures, from the instantons to their constituents - instanton-dyons - has recently lead to studies of their ensembles and very significant advances. Like instantons, they have fermionic zero modes, and their collectivization at sufficiently high density explains the chiral symmetry breaking transition. Unlike instantons, these objects have electric and magnetic charges. Simulations of the instanton-dyon ensembles have demonstrated that their back reaction on the Polyakov line modifies its potential and generates the deconfinement phase transition. For the $N_{c}=2$ gauge theory the transition is second order, for QCD-like theory with $N_{c}=2$ and two light quark flavors $N_{f}=2$ both transitions are weak crossovers at happening at about the same condition. Introduction of quark-flavor-dependent periodicity phases (imaginary chemical potentials) leads to drastic changes in both transitions. In particulaly, in the so called $Z\left(N_{c}\right)-Q C D$ model the deconfinement transforms to strong first order transition, while the chiral condensate does not disappear at all. The talk will also cover more detailed studies of correlations between the dyons, effective eta' mass and other screening masses.
\end{abstract}

\section{Introduction}

Since I participate in Lattice conference series intermittedly, I try to put in these few pages a very wide range of issues. Section 2 emphasizes topology-induced phenomena, such as chiral symmetry breaking, and technical-but-crucially-important limitations they pose for lattice simulations. Section 3 introduces the instanton-dyons and section 4 analytical and numerical studies of their ensembles. Those discussions are rather brief, for a bit more detailed discussion see 2016 proceedings [1] and original papers. Calculation of hadronic correlators using instanton-dyon ensembles, and the lesson followed, are summarized in section 5 .

Perhaps it is worth to remind lattice community about the "Gauge topology" workshop series. The first took place in August 2015 at Symons center, Stony Brook, the second in Nov.2016 at

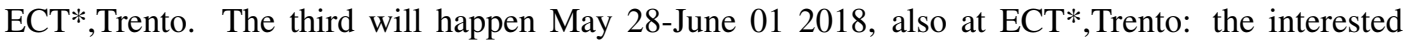
readers take a note.

\footnotetext{
^ The author emphasizes that this talk is based on works done in collaboration with R.Larsen, I.Zahed and Y.Liu.
} 


\section{Why is understanding of the gauge topology so important for lattice community?}

One general reason is that the topological solitons, once observed and studied on the lattice, can then be used to understand many other important phenomena. Some of them cannot be done in Euclidean time settings, requiring real time kinetics. E.g. the monopoles were long studies on the lattice, with a confirmation (see e.g. [2]) that they do Bose condensed below $T_{c}$. The monopoles not only explain confinement, but also were used to explain small viscosity (mean free path) [3] and strong jet quenching $[4,5]$ in quark-gluon plasma, especially near the transition temperature $T_{c}$.

Another reason is that understanding properties of topological objects on the lattice is in fact nacessary to answer many lattice questions, among them such basic ones as: "How large should be the lattices used in practice?"

In order to explain this point, let me briefly remind the history of chiral symmetry breaking. In a classic paper Nambu and Jona-Lasinio showed that an attraction in the scalar $\bar{q} q$ channel, if strong enough, can dynamically "gap" the surface of the Dirac sea. The origin of this interaction - claimed to be the origin of the mass of the "constinuent quarks", the nucleons and thus ourselves - was of course in 1961 completely unknown. In my paper [6], it was suggested that NJL attraction is nothing else but 't Hooft effective $2 N_{f}$ interaction induced by instantons. Two parameters of the NJL model has been substituted by another two: the total instanton-antiinstanton density $n \equiv 1 / R^{4} \sim 1 / \mathrm{fm}^{4}$ and their typical size $\rho \sim 1 / 3 \mathrm{fm}$. Of course, 't Hooft vertex does more than the NJL operator: in particular, it breaks the $U(1)_{A}$ symmetry.

Statistical mechanics of instanton ensemble, including 't Hooft interaction to all orders, known as the Interacting Instanton Liquid Model, has been developed and solved numerically in 1990's, for a review see [7]. Among other things, it introduced the notion of "collectivized zero mode zone", or $\mathrm{ZMZ}$ for short. The width of ZMZ is of the order of the typical matrix element of a "quark hopping matrix" made of overlaps of zero modes of neighboring instantons. Its magnitude is of the order of

$$
\Delta \lambda_{Z M Z} \sim \frac{\rho^{2}}{R^{3}} \sim 20 \mathrm{MeV}
$$

(for $\rho, R$ of the instanton ensemble mentioned above).

While these features and values of the parameters have been confirmed on the lattice many times, lattice community still had not appreciated its importance, and keeps rediscovering it. For example, Graz group had observed [8], that (i) most fluctuations in simulations come from few Dirac eigenmodes with eigenvalues $|\lambda|<\Delta \lambda_{Z M Z}$; (ii) their removal from propagators lead to significant $\sim 30-50 \%$ changes of hadronic masses; (iii) in a way that both the $S U\left(N_{f}\right)$ and $U(1) a$ chiral symmetries get restored.

Several plenary talks at this (and certainly earlier) lattice conferences discussed the requirements for the lattice sizes, assuming the smallest scale appearing in quark propagators is $m_{\pi} / 2$. This would indeed be true when quark masses go to zero, but not in the real world!. Indeed, $m_{\pi} / 2 \approx 70 \mathrm{MeV}$, significantly exceeding the ZMZ width $\Delta \lambda_{Z M Z}$ (which b.t.w. is essentially independent on the box size and quark masses). Multiple failures of chiral extrapolation fits to various quantities over the years attest to the same statement. It is known and published for at least two decades, yet the lesson has not yet been learned.

Another aspect at this issue can be explained as follows. The number of ZMZ eigenmodes is nothing but the mean number of instantons on the lattice, their density times the lattice volume. With current lattice spacings $a \sim .1 \mathrm{fm}$, the lattice volumes of the order of $(2 \mathrm{fm})^{4}$ may look sufficient: and yet they include on average only about $16=2^{4}$ instantons, or one instanton and one antiinstanton per direction. Not surprising, one finds large fluctuations and severe finite volume effects. Instanton 
liquid simulations were done with larger boxes, typically with 500 instantons. Sorry to say, (much more expensive) lattice simulations need such sizes of the box as well.

In conclusion, the gauge topology provides a serious additional argument in favor of the proposal, suggested by Luscher at this meeting, to use lattices as large as possible, even if the available resources would only be enough for a single or few configurations.

\section{Instanton-dyons}

The so called Polyakov line is used as a deconfinement order parameter, being nonzero at $T>T_{c}$. Interpreting this as existence of nonzero average $A_{0}$ field, one needs to modify all clasical solutions respectively. When such solutions were found in $1998[9,10]$ it has been realized that instantons get split into $N_{c}$ (number of colors) constituents, the selfdual instanton-dyons ${ }^{1}$, connected only by (invisible) Dirac strings. Since these objects have nonzero electric and magnetic charges and source Abelian (diagonal) massless gluons, the corresponding ensemble is an "instanton-dyon plasma", with long-range Coulomb-like forces between constituents.

The first application of the instanton-dyons were made soon after their discovery in the context of supersymmetric gluodynamics [11]. This paper solved a puzzling mismatch of the value of the gluino condensate, between the instanton-based and general supersymmetric evaluations of it.

Diakonov and collaborators (for review see [12] ) emphasized that, unlike the (topologically protected) instantons, the dyons interact directly with the holonomy field. They suggested that since such dyon (anti-dyon) become denser at low temperature, their back reaction may overcome perturbative holonomy potential and drive it to its confining value, leading to vanishing of the mean Polyakov line, or confinement. Specifically, Diakonov and collaborators focused on the self-dual sector $L, M$ and studied the one-loop contribution to the partition function [13]. The volume element of the moduli space was written in terms of dyons coordinates as a determinant of certain matrix $G$, to be referred to as Diakonov determinant. In a dilute limit it leads to Coulomb interactions between the dyons, but in the dense region it becomes strongly repulsive, till at certain density the moduli volume vanishes.

A semi-classical confining regime has been defined by Poppitz et al [14, 15] in a carefully devised setting of softly broken supersymmetric models. While the setting includes a compactification on a small circle, with weak coupling and an exponentially small density of dyons, the minimum at the confining holonomy value is induced by the repulsive interaction in the dyon-antidyon molecules (called bions by these authors). The crucial role of the supersymmetry is the cancellation of the perturbative Gross-Pisarski-Yaffe-Weiss (GPYW) [16] holonomy potential: as a result, in this setting there is no deconfined phase with trivial holonomy at all, unless supersymmetry is softly broken. Sulejmanpasic and myself [17] proposed a simple analytic model for the dyon ensemble with dyonantidyon "repulsive cores", and have shown how they may naturally induce confinement in dense enough dyonic ensemble.

Recent progress to be discussed below is related to studies of the instanton-dyon ensembles. We will focus on a series of papers devoted to high-density phase and mean field approximation [18-22] and on the direct numerical simulation of the dyon ensembles [23-27] in section 4.

Periodicity condition along the Matsubara circle can be defined with some arbitrary angles $\psi_{f}$ for quarks with the flavor $f$. As was determined by van Baal and collaborators, fermionic zero mode "hops" from one type of dyon to the next at certain critical values. The resulting rule is: it belogs to the dyon corresponding to the segment of the holonomy circle $v_{i}$ to which the periodicity phase belongs: $\mu_{i}<\psi_{f}<\mu_{i+1}$.

\footnotetext{
${ }^{1}$ They are called "instanton-monopoles" in applications to supersymmetric settings, e.g. by Khose et al and Unsal et al. Similar (but no idenical) objects were called the "instanton quarks" by Zhitnitsky et al.
} 
In physical QCD all quarks are fermions, so $\psi_{f}=\pi$ for all $f$. Therefore all $N_{f}$ quarks have zero modes bound to one and the same dyon type, the $L$-dyons. But one can introduce other arrangements of these phases. In particular, for $N_{c}=N_{f}$, the opposite extreme is the so called $Z\left(N_{c}\right) Q C D$, proposed in [28]. These authors suggested to imply imaginary chemical potentials in such a way, as to put the fermionic periodicity phases symmetrically around the circle. In the theory of the instanton-dyons this means that each dyon type has one - its "own" - quark flavor which binds to it.

\section{Instanton-dyon ensembles}

The first direct simulation of the instanton-dyon ensemble with dynamical fermions has been made by Faccioli and myself in [23]. The general setting follows the example of the "instanton liquid", it included the determinant' of the so called "hopping matrix", a part of the Dirac operator in the quasizero-mode sector. It has been done for $S U(2)$ color group and the number of fermions flavors $N_{f}=1,2,4$. Except in the last case, the chiral symmetry breaking phase transitions have been clearly observed, for dense enough dyon ensemble.

Larsen and myself [25] use direct numerical simulation of the instanton-dyon ensemble, both in the high-T dilute and low-T dense regime. Unlike the previous work, it uses classical dyon-antidyon interaction determined in Ref.[24]. The holonomy potential as a function of all parameters of the model is determined and minimized. At high density of the dyons their back reaction shifts the minimum to $v=1 / 2$, which is the confining value for $\mathrm{SU}(2)(\cos (\pi v)=0)$ : the confinement transition is thus generated. The self-consistent parameters of the ensemble, minimizing the free energy, is determined for each density. The transition is second order, as expected based on lattice results.

The next work of Larsen and myself [26] addressed the issue of chiral symmetry breaking in the $N_{c}=2$ theory with two light quark flavors $N_{f}=2$. The results for the mean Polyakov line are shown in Fig.1 (left) by blue filled circles as a function of the parameter $S$, the "instanton action", proportional to the log of the temperature

$$
S=\left(\frac{11 N_{c}}{3}-\frac{2 N_{f}}{3}\right) \log \left(\frac{T}{\Lambda}\right)
$$

So, larger $S$ at the r.h.s. of the figure correpond to high $T$, and thus to more dilute dyon ensemble, since densities contain $\exp \left(-S_{i}\right)$. The measure behavior of Polyakov line VEV in this theory indicates a smooth cross over transition.

This numerical simulations are done for dynamical quarks, with a partition function appended by the fermionic determinant evaluated in the zero mode approximation. Using two sizes of the system, with 64 and 128 dyons, we identify the finite-size effects in the eigenvalue distribution, and extrapolate to infinite size system. The location of the chiral transition temperature is defined both by extrapolation of the quark condensate, from below, and the so called "gaps" in the Dirac spectra, from above. The values of the quark condensate are shown by black triangles in the right side of Fig.1.

We do indeed observe, for this QCD-like SU(2) gauge theory with 2 flavors of light fundamental quarks, that the deconfinement and chiral symmetry restoration transitions occur about at the same dyon density, namely $S=7.5-8$ (see short vertical lines shown for convenience). Determination of the precise transition points is difficult since both transitions appear to be in this case just a smooth crossovers. By the way, the dyon actions are in the confined phase a half of it, so the action per dyon in the simulations is about $4 \hbar$. This four is the large parameter of the semiclassical approximation used.

The $Z\left(N_{c}\right)$ QCD has been studied in the mean field framework [22], by statistical simulations [27] and also by lattice simulations [29]. The first two papers consider the $N_{c}=N_{f}=2$ version of the 
theory, while the last one focus on the $N_{c}=N_{f}=3$. In the former case the set of phases are $\psi_{f}=0, \pi$, so one quark is a boson and one is a fermion. In the latter $\psi_{f}=\pi / 3, \pi,-\pi / 3$.

All these works find that the deconfinement transition in $Z\left(N_{c}\right)$ QCD strengthens significantly, as compared to the usual QCD with the same $N_{c}, N_{f}$. In place of a smooth crossover, in [22] find the second order transition, while our simulations [27] - the red squares at the left side of Fig.1 - and those on the lattice [29] both see strong jumps in the Polyakov line VEV, indicated strong first order transition.

All three studies see a non-zero chiral condensates in the studied region of densities: none of them find any indications for a chiral symmetry restoration. The values of the condensates for two quark flavors from our work [27] are shown by red squares and blue circles in the right side of Fig.1. We also found that the spectrum of the Dirac eigenvalues has a very specific "triangular" shape, characteristic of a single-flavor QCD. This explains why the $Z\left(N_{c}\right)$ QCD has much larger condensate than ordinary $\mathrm{QCD}$, at the same dyon density, and also why there is no tendedecy to restoration. As expected, all three works see that above the deconfinement transition there are no symmetry between flavors, so they have different chiral condensates, $\langle\bar{u} u>\neq<\bar{d} d>$. Also interesting that their difference is markedly smaller than one could expect from the difference in the dyon densities.
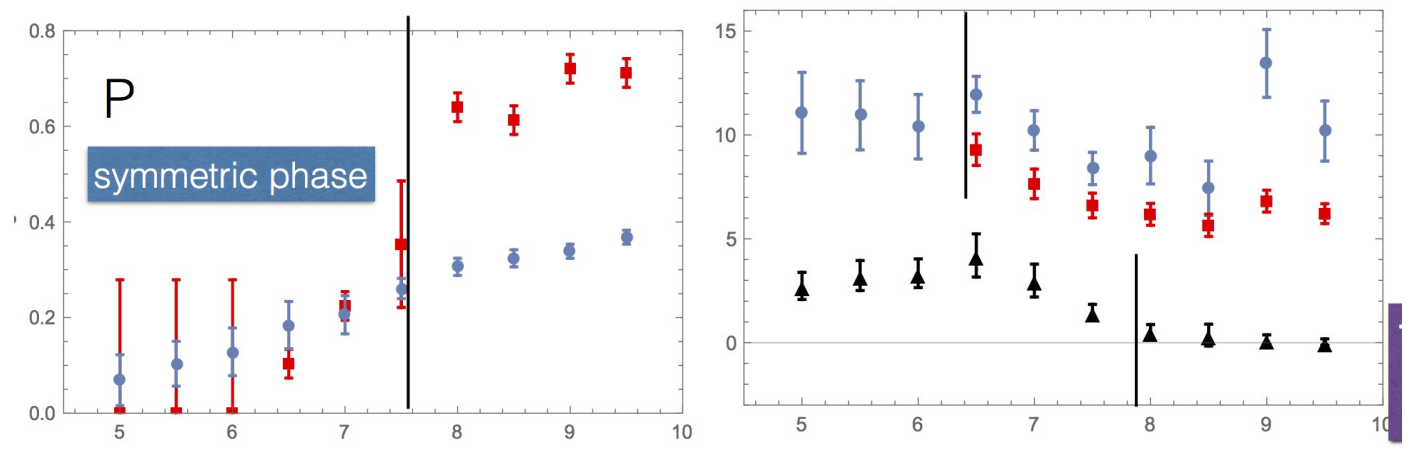

Figure 1. (left) The mean Polyakov line $\mathrm{P}$ versus the density parameter $S$. Red squares are for $Z_{2} Q C D$ while blue circles are for the usual QCD, both with $N_{c}=N_{f}=2$. (right) The quark condensate versus the density parameter $S$. Black triangles correspond to the usual QCD: and they display chiral symmetry restoration. Blue and red poins are for two flavor condensates of the $Z_{2} Q C D$ : to the left of vertical line there is a "symmetric phase" in which both types of dyons and condensates are the same.

\section{Hadronic point-to-point correlation functions and instanton-dyons}

Let us start this last section with a brief discussion of a more general question: At which temperatures the description of gauge topology in terms of the instanton-dyons is expected to work?

It is clear that that they should not be used at high enough $T$, because their whole existence is based on presence of non-zero VEV of the Polyakov line, which is absent at, say $T>400 \mathrm{MeV}$. And indeed, as we now know from recent lattice studies of the topological susceptibility and other considerations, at such high $T$ the gauge topology is well represented by a dilute instanton gas.

The instanton-dyon description is most useful near the deconfinement-chiral phase transition temperature, and this is what the discussion in the previous section was about.

At sufficiently low $T$ one, unfortunately, again has to abandon the instanton-dyon theory. One reason for that is that the Polyakov line VEV and the dyon fields both have scales $O(T)$, and as $T \rightarrow 0$ 
those become small compared to quantum scales related to $\Lambda_{Q C D}$ and thus unimportant. Another way to say it is as follows: as the size of the Matsubara time box $\beta=1 / T$ grows, the 3-dimensional objects - instanton-dyons - must strongly interfere with each other, in order to cancel each-other's fields in such a manner as to, eventually, reproducing the instantons, which are 4-dimensionally symmetric objects. These instantons also should have finite sizes and density as $T \rightarrow 0$. In other words, the $T \rightarrow 0$ limit is very singular for the instanton-dyon teory.

Now we formulate the main question of our paper [30]: Can the instanton-dyon theory, taken at its lowest end of temperatures $T \sim 100 \mathrm{MeV}$, reproduce known phenomenology of hadronic correlation functions?

The answer was affirmative, but with a nontrivial twist. It turned out, that it can only happen if the instanton-dyons of $M_{1} M_{2}$ types are strongly correlated with the $L$-type dyons, the only which have fermionic zero modes. These correlations are necessary to make these zero modes sufficiently well localized, and therefore strong enough, to reproduce splittings between the channels of the correct magnitude.

These splittings are shown in Fig.2. The left figure shows point-to-point correlators for four channels, normalized to those for free massless quarks (and thus all converging to 1 at small distances). Note rather symmetric splittings between "attractive" channels $P S, V$ when the non-perturbative effect is positive, and "repulsive" channels $A, S$. Note also that the $P S-S$ spliting, indicating violation of $U(1)_{a}$ chiral symmetry, is several times stronger than the $V-A$ splitting, indicating the violation of $S U\left(N_{f}\right)$ chiral symmetry. This fact, known phenomenologically for a long time, is one of the strongest indication of the important role of topology in hadronic spectroscopy.

These nice results, being in agreement with phenomenology and previous lattice studies, were not observed for a generic random instanton-dyon ensemble. Indeed, it only happens if the $M-L$ dyon correlations are sufficiently strong. The right figure indicates how we made the normalization of those in our ensemble: the so called $M L$ dyon correlation parameter has been tuned to one particular combination, the $V-A$ (vector-minus-axial) correlator. While being weaker than the $P S-S$ splitting, as we already mentioned, this one is rather accurately known, both from experiment (the shaded area, corresponding to ALEPH data on the spectral densities) and also from recent lattice work [32]) (small red and blue points). 

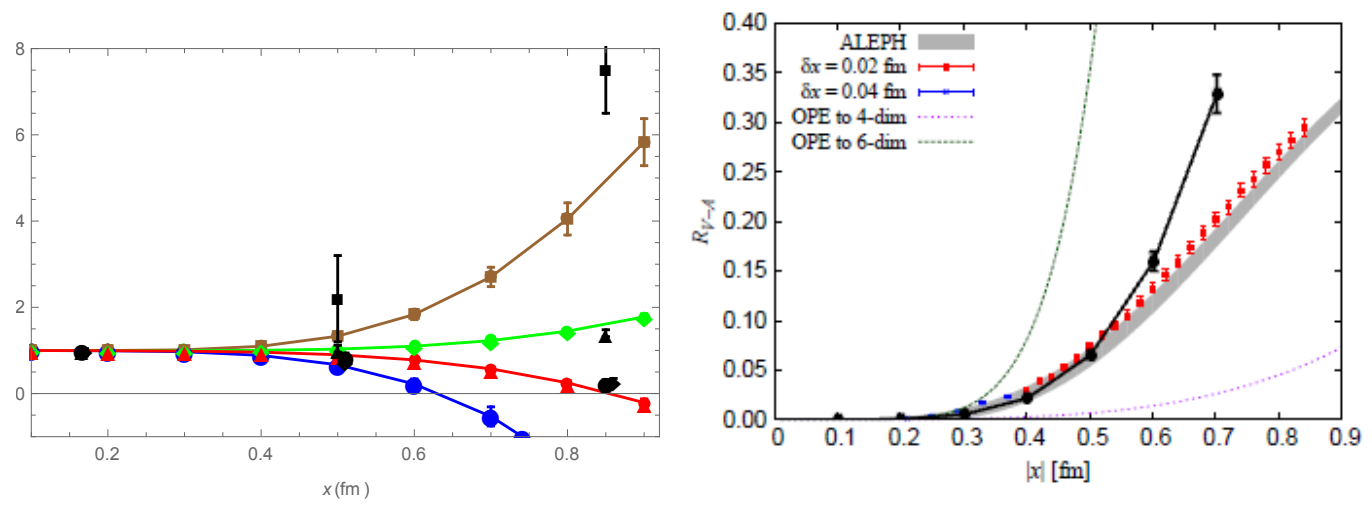

Figure 2. (Left figure): The colored points are our results for the normalized point-to-point correlators, for four channels: Pseudoscalar (Brown), Vector (Green), Axial vector (Red), and Scalar (Blue), top to bottom. The individual (black) points without lines are lattice data from [31], their symbols are the same as for our data. (Right figure) The normalized vector-minus-axial difference $(V(x)$ ? $A(x))$ versus the distance $\mathrm{x}(\mathrm{fm})$. The narrow shadowed region corresponds to ALEPH data, the red and blue dots correspond to the lattice data [32], for two lattice spacings indicated on the plot. Our results are shown by black points. 


\section{References}

[1] E. Shuryak, EPJ Web Conf. 137, 01018 (2017), 1610.08789

[2] A. D’Alessandro, M. D’Elia, E.V. Shuryak, Phys. Rev. D81, 094501 (2010), 1002 . 4161

[3] C. Ratti, E. Shuryak, Phys. Rev. D80, 034004 (2009), 0811.4174

[4] J. Xu, J. Liao, M. Gyulassy, Chin. Phys. Lett. 32, 092501 (2015), 1411.3673

[5] A. Ramamurti, E. Shuryak (2017), 1708.04254

[6] E.V. Shuryak, Nucl. Phys. B203, 93 (1982)

[7] T. Schäfer, E.V. Shuryak, Rev. Mod. Phys. 70, 323 (1998), hep-ph/9610451

[8] L.Ya. Glozman, C.B. Lang, M. Schrock, Acta Phys. Polon. Supp. 5, 1001 (2012), 1207.7323

[9] T.C. Kraan, P. van Baal, Phys. Lett. B435, 389 (1998), hep-th/9806034

[10] K.M. Lee, C.h. Lu, Phys. Rev. D58, 025011 (1998), hep-th/9802108

[11] N.M. Davies, T.J. Hollowood, V.V. Khoze, M.P. Mattis, Nucl. Phys. B559, 123 (1999), hep-th/9905015

[12] D. Diakonov, Nucl. Phys. Proc. Suppl. 195, 5 (2009), 0906.2456

[13] D. Diakonov, N. Gromov, V. Petrov, S. Slizovskiy, Phys. Rev. D70, 036003 (2004), hep-th/0404042

[14] E. Poppitz, M. Unsal, JHEP 07, 082 (2011), 1105.3969

[15] E. Poppitz, T. Schäfer, M. Unsal, JHEP 10, 115 (2012), 1205.0290

[16] D.J. Gross, R.D. Pisarski, L.G. Yaffe, Rev. Mod. Phys. 53, 43 (1981)

[17] E. Shuryak, T. Sulejmanpasic, Phys. Lett. B726, 257 (2013), 1305. 0796

[18] Y. Liu, E. Shuryak, I. Zahed, Phys. Rev. D92, 085006 (2015), 1503.03058

[19] Y. Liu, E. Shuryak, I. Zahed, Phys. Rev. D92, 085007 (2015), 1503.09148

[20] Y. Liu, E. Shuryak, I. Zahed, Phys. Rev. D94, 105011 (2016), 1606. 07009

[21] Y. Liu, E. Shuryak, I. Zahed, Phys. Rev. D94, 105012 (2016), 1605 . 07584

[22] Y. Liu, E. Shuryak, I. Zahed, Phys. Rev. D94, 105013 (2016), 1606. 02996

[23] P. Faccioli, E. Shuryak, Phys. Rev. D87, 074009 (2013), 1301.2523

[24] R. Larsen, E. Shuryak, Nucl. Phys. A950, 110 (2016), 1408.6563

[25] R. Larsen, E. Shuryak, Phys. Rev. D92, 094022 (2015), 1504.03341

[26] R. Larsen, E. Shuryak, Phys. Rev. D93, 054029 (2016), 1511.02237

[27] R. Larsen, E. Shuryak, Phys. Rev. D94, 094009 (2016), 1605.07474

[28] H. Kouno, Y. Sakai, T. Makiyama, K. Tokunaga, T. Sasaki, M. Yahiro, J. Phys. G39, 085010 (2012)

[29] T. Misumi, T. Iritani, E. Itou, PoS LATTICE2015, 152 (2016), 1510.07227

[30] R. Larsen, E. Shuryak, Phys. Rev. D96, 034508 (2017), 1705.04707

[31] M.C. Chu, J.M. Grandy, S. Huang, J.W. Negele, Phys. Rev. Lett. 70, 255 (1993), hep-lat/9211019

[32] M. Tomii, G. Cossu, B. Fahy, H. Fukaya, S. Hashimoto, T. Kaneko, J. Noaki (JLQCD), Phys. Rev. D96, 054511 (2017), 1703.06249 